\title{
Principle for the Realization of Dual-Orthogonal Linearly Polarized Antennas for UWB Technique
}

\author{
Grzegorz Adamiuk, Mario Pauli, and Thomas Zwick \\ Institute of High Frequency Techniques and Electronics (IHE), Karlsruhe Institute of Technology (KIT), Kaiserstraße 12, \\ 76131 Karlsruhe, Germany \\ Correspondence should be addressed to Grzegorz Adamiuk, grzegorz.adamiuk@ihe.uka.de
}

Received 20 May 2011; Revised 26 July 2011; Accepted 27 July 2011

Academic Editor: Zhongxiang Q. Shen

Copyright ( $\odot 2011$ Grzegorz Adamiuk et al. This is an open access article distributed under the Creative Commons Attribution License, which permits unrestricted use, distribution, and reproduction in any medium, provided the original work is properly cited.

\begin{abstract}
A concept of an array configuration for an ultrawideband suppression of the cross-polarization is presented. The method is explained in detail, and a mathematical description of the principle is given. It is shown that the presented configuration is convenient for the development of very broad band, dual-orthogonal, linearly polarized antennas with high polarization purity. The investigated configuration shows a high decoupling of the orthogonal ports and is capable for antennas with a main beam direction perpendicular to the substrate surface, that is, for a planar design. The phase center of the antenna configuration remains fixed at one single point over the complete desired frequency range, allowing a minimum dispersion of the radiated signal. The influence of nonidealities in the feeding network on the polarization purity is investigated. The presented method introduces a superior possibility of an extension of typical UWB technique to fully polarized systems, which improves significantly performance in, for example, UWB-MIMO or UWB-Radar.
\end{abstract}

\section{Introduction}

The ultrawideband (UWB) mask as it is defined by the FCC [1] allows the licence-free usage of the spectrum between $3.1 \mathrm{GHz}$ and $10.6 \mathrm{GHz}$ with the maximal power spectral density of $-41.3 \mathrm{dBm} / \mathrm{MHz}$. This bandwidth opens many possibilities, not only in communication systems with potentially high data rates but also in localization and radar systems, where the accuracy or resolution is directly proportional to the bandwidth. On the other hand, the large bandwidth is challenging regarding the antenna design. This is especially true for dual polarized antennas. The latest research in the ultrawideband technology shows an increasing demand on ultrawideband dual polarized antenna elements [2]. For many applications, polarization diversity is of big importance. In case of single polarization, there could be a degradation of the performance if transmit and receive antenna are cross-polarized, especially in a line-of-sight environment. Polarization diversity can also enhance the performance of radar and imaging systems [3-5] and is nearly indispensable in the MIMO technique.
In literature, only few dual-orthogonal polarized ultra wideband antenna concepts can be found. Most of them radiate the electromagnetic wave over a very widebandwidth using the traveling wave principle like the well-known quad-ridged horn antenna [6], the dielectric rod antenna [7] or the tapered slot (a.k.a. vivaldi) antenna [8]. However, such antennas are generally bulky and difficult to integrate into any device. Furthermore, only few antennas offer a polarization purity of better than $20 \mathrm{~dB}$ over the bandwidth of several GHz. All antennas based on traveling wave principle need generally a $3 \mathrm{D}$ structure in order to perform correctly. Such design makes an integration of the radiators into any compact device difficult. For this reason, a planar solution with a high cross-polarization suppression has been investigated to assure a wideband radiation of two orthogonal linear polarizations. An important demand on the antenna is a stable phase center of radiation, which location is constant over the very widebandwidth. This guarantees small distortion of the radiated pulse in pulsebased UWB systems. The phase centers should possess the same position for both polarizations, what assures the 
same radiation conditions independent of the polarization mode. For the integration of the radiators into a device, the radiation pattern which is perpendicular to the antenna surface is advantageous. The main beam direction should be constant over the frequency range. Similar beamwidths in the $E$-plane and in the $H$-plane allow for the illumination of the same space independent of the polarization. This paper presents a principle of an array configuration for the realization of ultrawideband dual-orthogonal, linearly polarized antennas meeting the above-mentioned requirements. The array configuration for suppression of the cross-polarization in a certain angular region is described. The principle introduces simultaneously a method for stabilization of the movement of the phase center over the frequency. In the following, a mathematical description of the radiation characteristics is given, and the performance of the array configuration is evaluated. In the last part of the paper the influence of nonidealities (amplitude and phase balance) on the overall performance of the proposed configuration is investigated.

\section{Array Configuration}

First, a single, arbitrary, planar antenna element, marked in Figure 1(a) as a blue surface, is considered. The target polarization is linear and conforms with $z$-axis. The radiated electrical field vector $E$ (solid arrow) is oriented arbitrarily. Firstly, the orientation in the $y z$-plane is considered. The radiated $E$-field vector is distributed into copolarized (dashed arrow) and cross-polarized (dotted arrow). The desired linear polarization is parallel to the $z$-axis and can be easily achieved by a rotation of the antenna. However, this is not applicable in practice since the polarization may turn with frequency. Furthermore, the rotation of the antenna is not a solution in the case of an unintended elliptical polarization. To overcome these problems, a second mirrored antenna is used, as shown in Figure 1(c). The configuration is symmetrical to the $y$-axis. If both antennas are fed by a signal which is identical in amplitude and phase, the radiated polarization (solid arrow) from both elements is also symmetrical with espect to the $y$-axis. This means that the cross-polarization components (dotted arrows) are identical in amplitude and phase and the co-polarization components are identical in amplitude but oriented in the opposite direction. This results in a cancelation of the desired copolarization. To overcome this drawback, a differential feeding of the antennas is employed, which is shown schematically in Figure 1(d). It can be noted that through the implementation of the differential feeding the cross-polarization vectors are oriented in opposite direction and therefore interfere destructively, whereas the co-polarization ones are in the same direction and interfere constructively. Such configuration results in the radiation of the desired linearly polarized $E$-field parallel to the $z$-axis. It is evident that the polarization is not depending on the electromagnetic characteristics of the single antenna but only on the mechanical arrangement of the single antenna elements within the array configuration. Noticeable is also that for the main beam direction ( $x$-axis) the principle of cancellation of

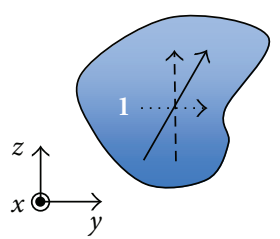

(a) Single element

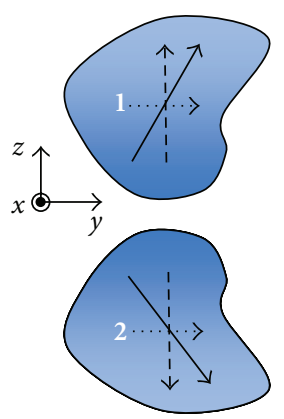

(c) Antenna array with mirrored elements

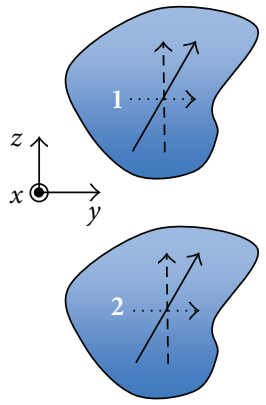

(b) Standard antenna array

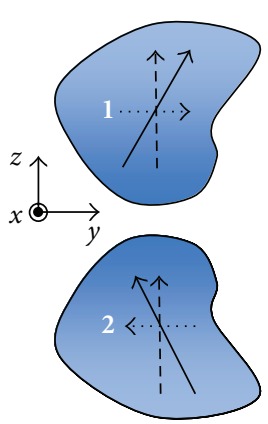

(d) Antenna array with mirrored, differentially fed elements

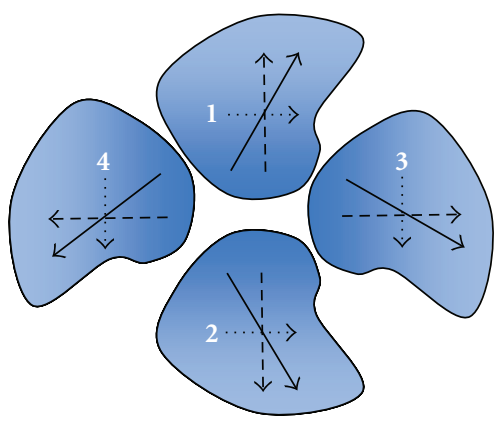

(e) Dual-orthogonal, linear polarized antenna array with mirrored, differentially fed elements
FIgURE 1: Various antenna array configurations and their influence on the polarization of the radiated wave.

cross-polarization with simultaneous enhancement of copolarization is independent of the wavelength. Hence, it is suitable for UWB technique.

To obtain two linear orthogonal polarizations, the antenna array has to be upgraded by two additional elements rotated by $90^{\circ}$ as shown in Figure 1(e). If both subarrays are operated independently, two linear orthogonal polarizations can be radiated independently.

Depending on the antenna type, the position of the phase center can be a function of the frequency, which results in an unwanted dispersion of the radiated pulse [9]. This variation of the phase center is schematically shown in Figure 2(a), where the phase center at a certain frequency $f_{i}$ is represented by the black dot. Within an antenna array with two elements the phase center is always located in the middle between the 


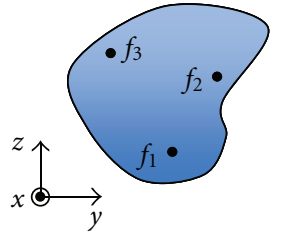

(a) Single element

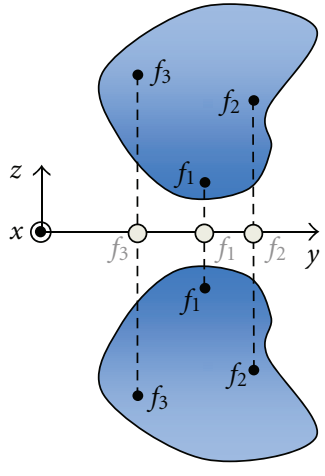

(b) Array with axissymmetrical elements
Figure 2: Variation of the phase center (black dot) of the radiator at different frequencies $f_{i}$.

phase centers of the two elements (regarding the main beam direction). Thus, in an antenna array with a symmetrical arrangement of the second radiator, the variation of the phase center is limited to the symmetry axis (here $y$-axis), what is shown in Figure 2(b) [10]. This results in a partial compensation of the dispersion characteristics of the antenna. This principle is valid as long as the antennas have identical electromagnetical properties and do not couple mutually. If the elements have a phase center that is only varying along one axis, with the proposed arrangement the phase center can be fixed at one single point over a wide frequency range. If a second antenna pair which is rotated around this point is used, a dual-polarized radiator with an identical, frequency-independent phase center for both polarizations is obtained.

Similar configurations have been shown in the literature $[11,12]$. The authors concentrated, however, on narrow band antennas. The independency of the principle of the frequency and the advantages regarding the stabilization of the phase center have not been fully revealed.

\section{Mathematical Description of Radiation}

In the following, a mathematical model for the radiation properties of a mirrored, differential-fed antenna array is derived. The antenna and the corresponding coordinate system is shown in Figure 3(a). The antenna placed in the upper hemisphere $(z>0)$ is indexed as antenna " 1 " and the one in the lower hemisphere $(z<0)$ as antenna " -1 ". The feeding voltages of both radiators are differential to each other and normalized to the input voltage. Hence, the feeding voltage can be described by

$$
\underline{U}_{1}=\frac{1}{\sqrt{2}}, \quad \underline{U}_{-1}=\frac{1}{\sqrt{2}} e^{j \pi} .
$$

The transfer function $\underline{\mathbf{H}}_{\mathrm{Ar}}(\theta, \psi)$ of the array configuration can be determined as follows

$$
\underline{\mathbf{H}}_{\mathrm{Ar}}(\theta, \psi)=\sum_{i} \underline{\mathbf{H}}_{i}(\theta, \psi) \cdot \underline{\boldsymbol{\Phi}}_{i}(\theta, \psi),
$$

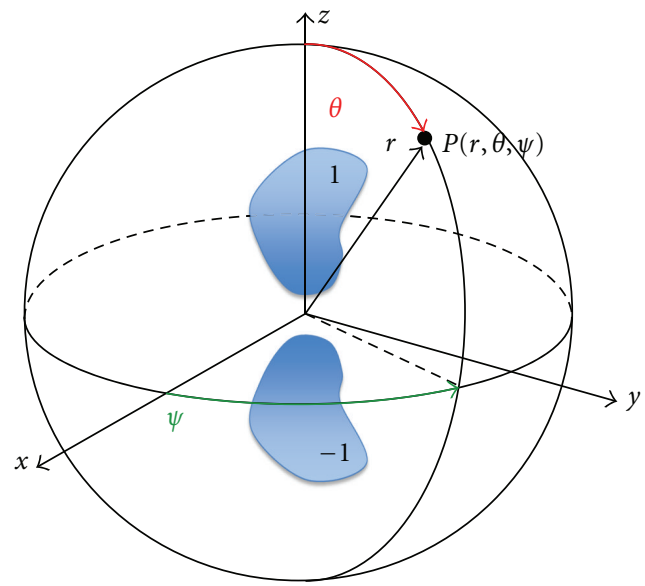

(a) Mirrored antenna array elements in the coordinate system

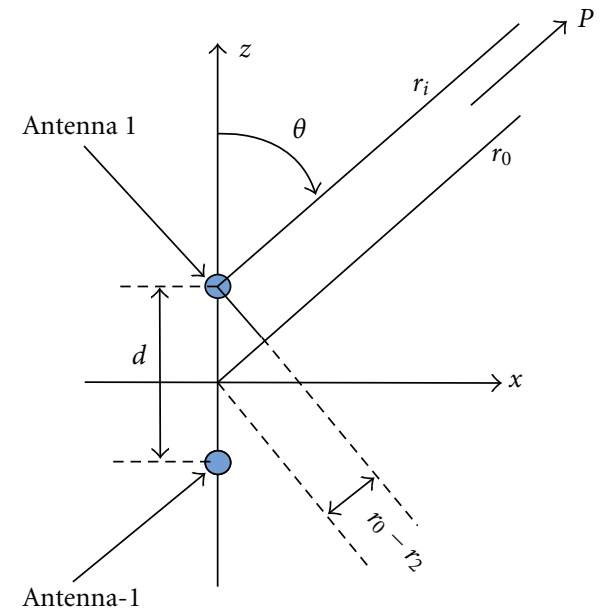

(b) Configuration for the determination of the array weighting factor $\underline{\Phi}_{i}(\theta, \psi)$

Figure 3: Coordinate system and configuration for the determination of the array transfer function $\underline{\mathbf{H}}_{\mathrm{Ar}}(\theta, \psi)$.

where $\underline{\mathbf{H}}_{i}(\theta, \psi)$ is the complex transfer function of the single element. $\underline{\Phi}_{i}(\theta, \psi)$ is a weighting factor which is derived from the definition of a typical array factor and is defined in the following formula:

$$
\begin{aligned}
\underline{\Phi}_{i}(\theta, \psi) & =U_{i} e^{-j \beta_{0}\left(r_{0}-r_{i}\right)}, \\
r_{0}-r_{i} & =i \frac{d}{2} \cos \theta, \\
\beta_{0} & =\frac{2 \pi}{\lambda_{0}},
\end{aligned}
$$

where $r_{i}$ represents the distance between the observation point $P$ and the base point of the coordinate system, $d$ is the distance between the two antennas (cf. Figure 3(b)), and $U_{i}$ is the amplitude of the feeding voltage. Combining (2)-(5), the transfer function of the array $\underline{\mathbf{H}}_{\mathrm{Ar}}(\theta, \psi)$ yields

$$
\begin{aligned}
\underline{\mathbf{H}}_{\mathrm{Ar}}(\theta, \psi)= & \underline{\mathbf{H}}_{-1}(\theta, \psi) \cdot \underline{U}_{-1} e^{-j \beta_{0}(-1)(d / 2) \cos \theta} \\
& +\underline{\mathbf{H}}_{1}(\theta, \psi) \cdot \underline{U}_{1} e^{-j \beta_{0}(1)(d / 2) \cos \theta},
\end{aligned}
$$


where $\underline{\mathbf{H}}_{1}$ and $\underline{\mathbf{H}}_{-1}$ are complex transfer functions of the corresponding antennas. After simplification, rearrangement with the exploitation of the geometrical symmetry of $\underline{\mathbf{H}}_{1}$ and $\underline{\mathbf{H}}_{-1}$, (6) can be written as functions of the polarization components

$$
\begin{aligned}
\underline{\mathbf{H}}_{\mathrm{Ar}}(\theta, \psi)= & {\left[\begin{array}{c}
\underline{H}_{1, \theta}(-\theta, \psi) \mathbf{u}_{\theta} \\
-\underline{H}_{1, \psi}(-\theta, \psi) \mathbf{u}_{\psi}
\end{array}\right] \cdot \frac{1}{\sqrt{2}} e^{j(1 / 2) \beta_{0} d \cos \theta} } \\
& +\left[\begin{array}{c}
\underline{H}_{1, \theta}(\theta, \psi) \mathbf{u}_{\theta} \\
\underline{H}_{1, \psi}(\theta, \psi) \mathbf{u}_{\psi}
\end{array}\right] \cdot \frac{1}{\sqrt{2}} e^{-j(1 / 2) \beta_{0} d \cos \theta},
\end{aligned}
$$

where $\mathbf{u}_{\theta}$ represents in this case the co-polarization unit vectors, and $\mathbf{u}_{\psi}$ represents the cross-polarization unit vector. It can be observed that the copolarized components $\mathbf{u}_{\theta}$ have the same leading sign, and the cross-polarized components $\mathbf{u}_{\psi}$ have opposite leading signs of the respective summands in the transfer function $\underline{\mathbf{H}}$. This results in constructive and destructive interference of co- and cross-polarization, respectively.

To investigate the influence of the antenna arrangement on the corresponding components $\mathbf{H}_{\mathrm{Ar}, \theta}(\theta, \psi)$ (co-pol) and $\mathbf{H}_{\mathrm{Ar}, \psi}(\theta, \psi)(x-\mathrm{pol})$, in the following, isotropic radiators are considered for the calculation of the transfer function $\underline{\mathbf{H}}_{\mathrm{Ar}}(\theta, \psi)$. One receives the array factor $\underline{\mathbf{A F}}$, which represents the radiation characteristics independent of the used elements. Writing (7) without the complex transfer functions of the elements $\underline{\mathbf{H}}_{1}$ and $\underline{\mathbf{H}}_{-1}$, the array factor $\underline{\mathrm{AF}}$ is obtained

$$
\begin{aligned}
\underline{\mathbf{A F}}(\theta, \psi)= & {\left[\begin{array}{c}
\mathbf{u}_{\theta} \\
-\mathbf{u}_{\psi}
\end{array}\right] \cdot \frac{1}{\sqrt{2}} e^{j(1 / 2) \beta_{0} d \cos \theta} } \\
& +\left[\begin{array}{c}
\mathbf{u}_{\theta} \\
\mathbf{u}_{\psi}
\end{array}\right] \cdot \frac{1}{\sqrt{2}} e^{-j(1 / 2) \beta_{0} d \cos \theta} .
\end{aligned}
$$

In case of two differentially fed antennas arranged symmetrically to the $y$-axis, the array factors for co-polarization $\left(\underline{\mathbf{A F}}_{\theta}\right)$ and cross-polarization $\left(\underline{\mathbf{A F}}_{\psi}\right)$ direction can be written as

$$
\begin{aligned}
& |\underline{\mathbf{A F}}(\theta, \psi)|=\left|\sqrt{2} \cos \left(\frac{1}{2} \beta_{0} d \cos \theta\right)\right|, \\
& \left|\underline{\mathbf{A F}}_{\psi}(\theta, \psi)\right|=\left|\sqrt{2} \sin \left(\frac{1}{2} \beta_{0} d \cos \theta\right)\right| .
\end{aligned}
$$

\section{Evaluation of the Radiation Characteristic of the Array}

In this section, the influence of the previously described antenna arrangement on the polarimetric radiation characteristics is evaluated. The array is arranged symmetrically to the $y$-axis, and the co- and cross-polarized radiation properties are described by (9) and (10), respectively. It can be seen in these equations that the array factors depend on the frequency, the distance between the antenna elements $d$ and the angle $\theta$, that is, the observation angle (cf. Figure 3(b)). This means that in some angular regions the co-polarization is enhanced, and the cross-polarization is suppressed and vice versa. This has to be taken into account in the antenna design. Noticeable is lack of the dependency on the angle $\psi$. It means that the radiation properties of the described array are projected with the same quality on the whole $\psi$-plane for certain angle $\theta$.

The array factors $\left|\mathrm{AF}_{\theta}\right|$ and $\left|\mathrm{AF}_{\psi}\right|$ are shown in Figure 4(a) for an electrical distance $d_{\mathrm{el}}=d / \lambda_{0}=0.5$. In the angular region between approximately $60^{\circ}$ and $120^{\circ}$, the $\theta$-component is amplified and the $\psi$-component is suppressed effectively. The maximum gain is $3 \mathrm{~dB}$ at an angle of $\theta=90^{\circ}$. At this angle, the cross-polarization is nearly completely suppressed. The angular dependencies of the array factors for electrical distances of $d_{\mathrm{el}}=1$ and $d_{\mathrm{el}}=1.5$ are shown in Figures 4(b) and 4(c), respectively. With increasing distance between the elements, the beams become narrower and grating lobes occur. In the main beam direction $\left(\theta=90^{\circ}\right)$, the maximum of $\left|\underline{A F}_{\theta}\right|$ and the minimum of $\left|\mathrm{AF}_{\psi}\right|$ are independent of the electrical distance between the antenna elements. In this region, the $\theta$-polarization is amplified and the $\psi$-polarization is suppressed independent of the frequency.

Figure 5 shows the mean array factor of the $\theta$ - and the $\psi$ component averaged over a bandwidth between $3.1 \mathrm{GHz}$ and $10.6 \mathrm{GHz}$. An effective suppression of the cross-polarization $\left(\left|\underline{A F}_{\psi}\right|\right)$ and an amplification of the co-polarization $\left(\left|\underline{A F}_{\theta}\right|\right)$ can be observed for the angles $75^{\circ}<\theta<105^{\circ}$. At $90^{\circ}$, the amplification of the $\theta$-component is $3 \mathrm{~dB}$, and the suppression of the $\psi$-component is maximal. The maximal amplification of the cross-polarization is approximately $1.9 \mathrm{~dB}$ at $60^{\circ}$ and $120^{\circ}$. It does not reach the value of $3 \mathrm{~dB}$ due to the angular movement of the maximum of the cross polarized beams over the frequency.

The diagrams in Figures 4 and 5 have been verified by the authors in terms of simulations and measurements for the monopolarized radiator. In the following section, a measurement verification for the dual-polarized array is presented.

\section{Measurement Verification}

The antenna for the verification is based on a well-known elliptical antenna, also known as diamond-shaped antenna $[10,13]$. The antenna is able to radiate over a very large bandwidth and possesses strong cross-polarization components in the $H$-plane. Hence, the properties of the radiator are convenient for the verification of the principle. The prototypes of the radiators presented in this paper are relatively bulky and are chosen only for verification purposes, due to their radiation properties. For compact realizations with stable radiation patterns over frequency and high polarization decoupling, the reader is referred to other works of the authors [14-17].

The prototype of the single radiator is presented in Figures $6(\mathrm{a})$ and $6(\mathrm{~b})$. The radiating element consists of an elliptical opening in the ground plane in which an elliptical metallization is incorporated. The antenna is fed at the slot between the inner ellipse and the ground plane. As a feeding network, a microstrip line with tapering is used. 

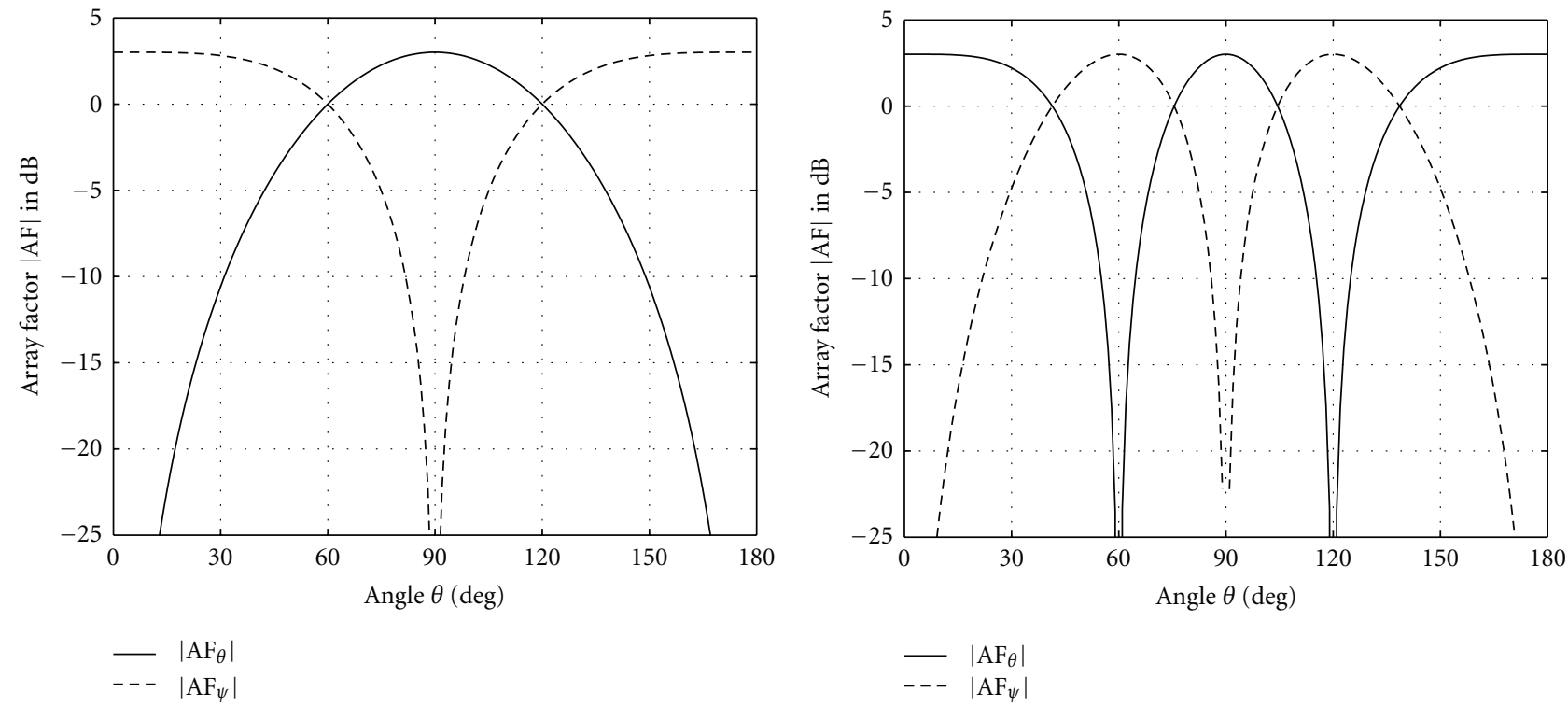

(a) $d_{\mathrm{el}}=0.5$

(b) $d_{\mathrm{el}}=1$

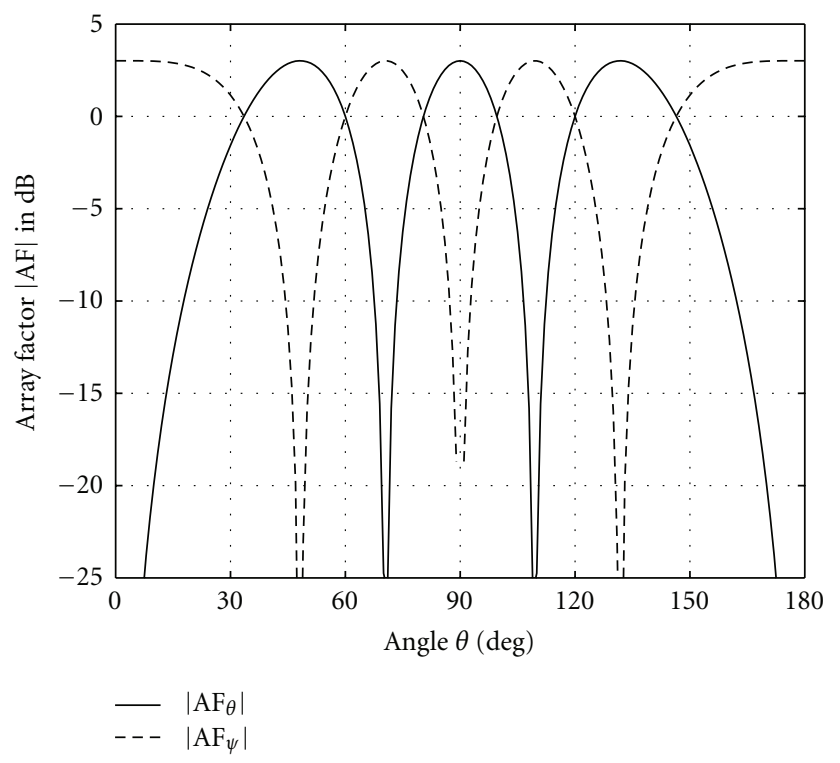

(c) $d_{\mathrm{el}}=1.5$

Figure 4: Dependency of the array factors $|\underline{\mathrm{AF}}|$ on the electrical distance $d_{\mathrm{el}}$ between the elements over $\theta$.

The dual-polarized array consisting of two pairs (four elements) of the previously presented antenna is shown in Figures $6(\mathrm{c})$ and $6(\mathrm{~d})$. The elements are pairwise symmetrical, and the symmetry points of both pairs are the same. As a feeding network also microstrip line is used. Since both elements of the single pair must be excited at the same time, a power divider must be applied. The outputs of the dividers are tapered and connected to the feeding points of the respective radiators. In order to be able to drive both polarizations independently, two feeding networks, each for one polarization, are applied.

The measured gain for the co-polarization in the $H$-plane for the single antenna and for the dual-polarized radiator (port 1) is shown in Figures 7(a) and 7(b), respectively.
In both cases, two beams at $0^{\circ}$ and $180^{\circ}$ are present, which yield an orientation perpendicular to the substrate. The similar widths of the beams are expected, since the aperture of the radiator is not extended in the $H$-plane. Noticeable is higher gain of the dual-polarized array, which results from the application of two elements, which extend the aperture along the $E$-plane. For the comparison, the measured results of the gain for the $E$-plane, co-polarization are shown in Figures 7(c) and 7(d), for the single element, and array, respectively. For the single element a not symmetrical radiation pattern with respect to $0^{\circ}$ over frequency is observed. This is a result of not symmetrical antenna geometry along this plane. Due to extension of the radiator to an array, a symmetrization of the current distribution 


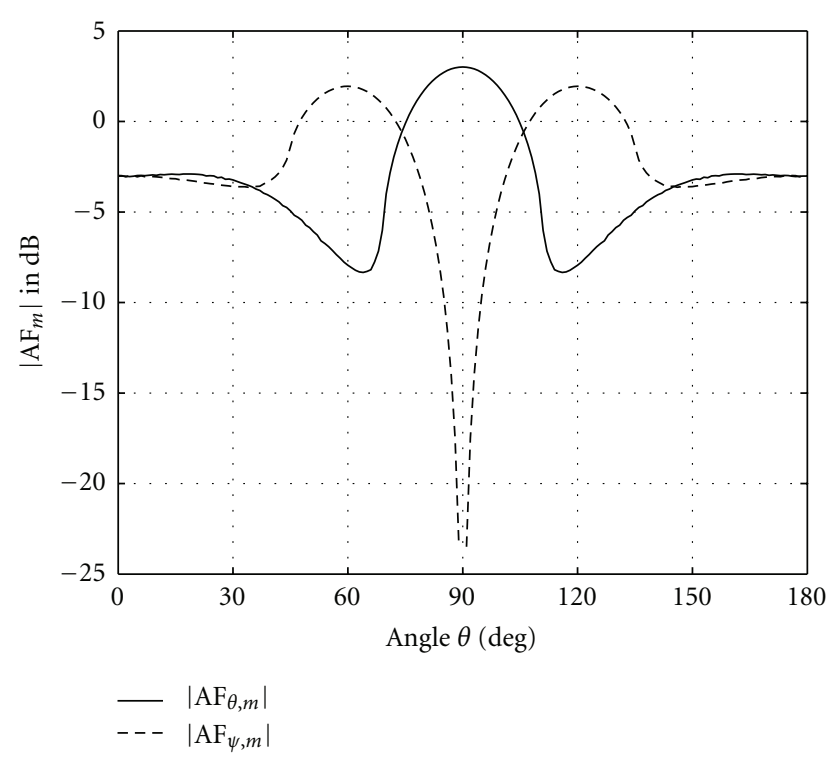

Figure 5: Mean array factor $\left|\underline{\mathrm{AF}}_{m}\right|$ in dependency of $\theta$.

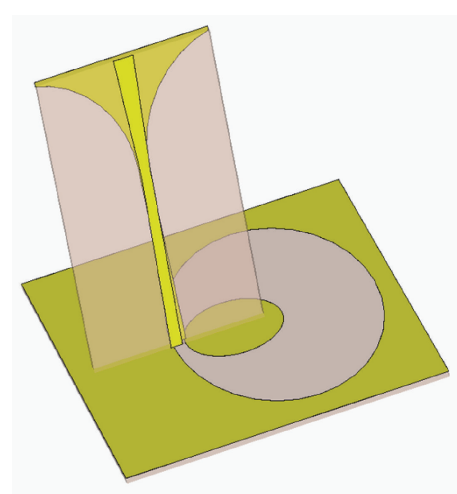

(a) Single antenna, model [10]

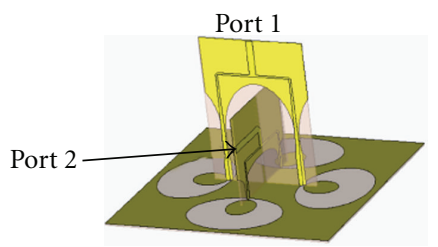

(c) Array, model

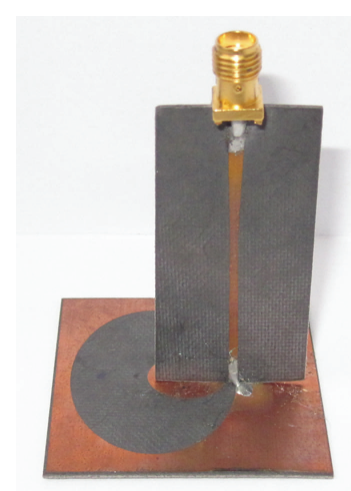

(b) Single antenna, prototype

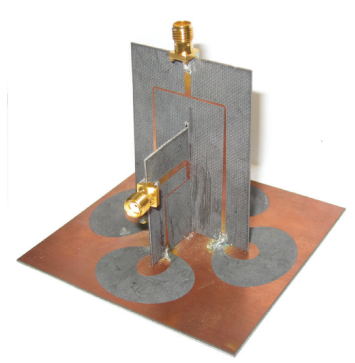

(d) Array, prototype
FIGURE 6: Single element and dual-orthogonal, linearly polarized, differentially fed antenna array for the verification.

along the E-plane is achieved; hence, the radiation pattern is more symmetric. Outside of the main beam direction $\left(0^{\circ}\right)$, side lobes are present, which are result of the relatively high distance between the elements. Due to previously mentioned extension of the aperture in the E-plane, a narrower beam is observed in the $E$-plane than in the $H$ plane. With the measurements, it has been shown that the array is able to enhance the co-polarization. For the full verification of the principle, a destructive influence of the configuration on the cross-polarization has to be included.

In Figures 8(a) and 8(b), the measured gains in the crosspolarization, $H$-plane are shown for single element and array, respectively (please note the scale). In the case of single element-four strong cross-polarized beams are present. These are extensively suppressed in the case of the array. Some stronger cross-polarized components in the angular region $|\theta|>90^{\circ}$ are a result of radiation from the feeding network of the array and can be significantly suppressed by shielding or compact realization as shown by the authors, for example, in $[14,15]$. For the completeness in Figure 8, the measurement results of single antenna and array for the $E$-plane, cross-polarization are shown. No significant negative effects considering the polarization purity are observed.

A further advantage of the presented antenna configuration is the high decoupling between the pairs of ports for orthogonal polarizations. This occurs due to the annihilation of the electric fields at the ports for orthogonal polarizations. This effect is described and verified by the measurements in $[14,15]$. In these publications, an important issue of miniaturization of the antenna array basing on the introduced principle is also presented.

It can be concluded that in certain angular regions, the cross-polarization can be successfully suppressed independent of the frequency. The size of the useful region is larger with decreasing frequency and distance between the elements. Hence, for the given frequency range, the distance between the mirrored elements should be kept small in terms of wavelengths. The concepts for the compact design of the radiator basing on the described principle are described by the authors in $[14,15]$. Due to the same aperture size of the cited antennas in the $E$ - and $H$-planes, the radiators possess nearly the same beamwidths in the respective planes over the frequency range from approximately $3 \mathrm{GHz}$ to $11 \mathrm{GHz}$. This introduces a significant advantage for the dual-polarized antennas, since the same space is illuminated independent on the polarization. The examples from the literature $[14,15]$ show an example of practical implementation of the heredescribed principle in the compact dual-orthogonal, linearly polarized UWB antennas.

The principle is applicable for nearly all types of radiators. However, it has to be kept in mind, that prior to the design of the antenna array and the selection of the symmetry plane an intensive investigation of the radiation characteristics of the single element has to be performed. This knowledge is indispensable for the proper arrangement of the elements and successful suppression of the crosspolarization.

\section{Requirements on the Feeding Network}

In the theoretical investigation, the differential feeding of the antennas was considered as ideal, that is, the signals feeding single elements were exactly equal in amplitude, and the phase shift was exactly $180^{\circ}$. However, this condition may not be met in reality due to the tolerances in the manufacturing process or a nonideal design of the feeding network. Hence, 


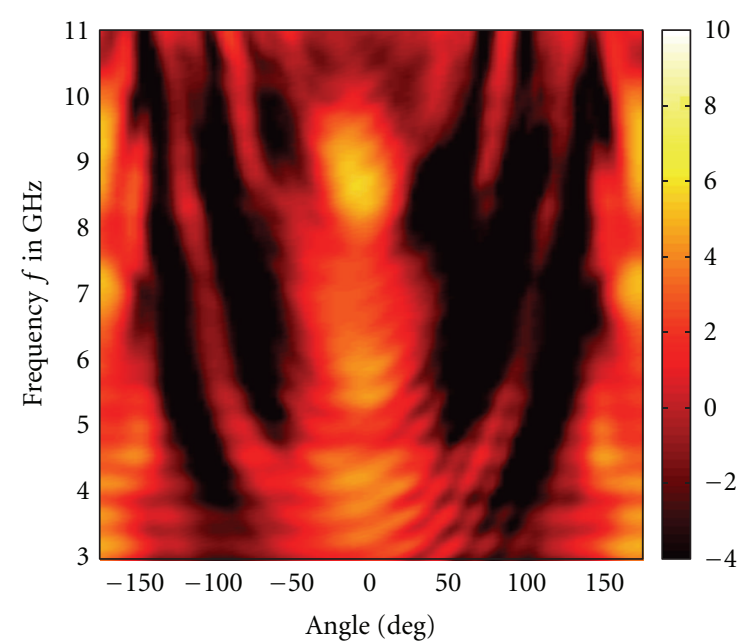

(a) Single antenna, $H$-plane

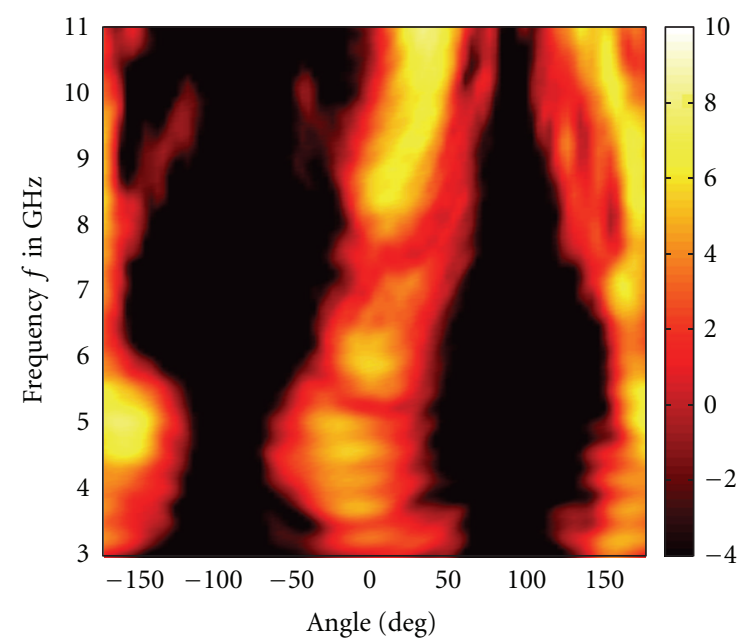

(c) Single antenna, E-plane

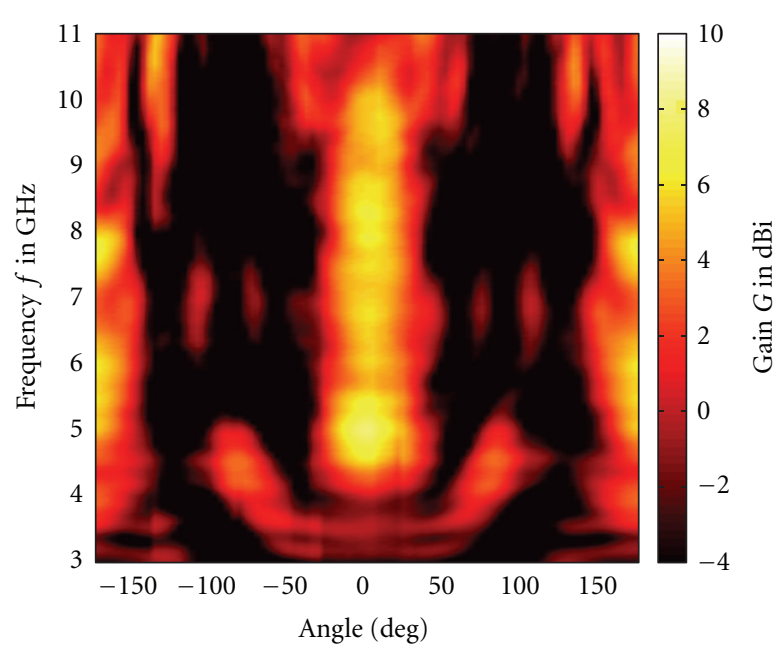

(b) Array, $H$-plane

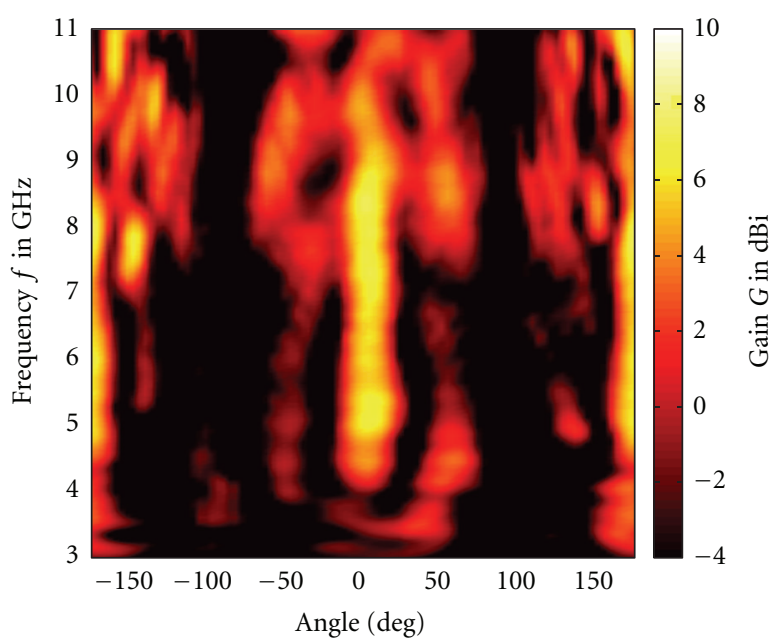

(d) Array, E-plane

Figure 7: Measured gain of the single element (Figure 6(b)) and array (Figure 6(d)) in the co-polarization, $E$ - and $H$-plane over the frequency.

the influence of imperfect feeding signals on the antenna performance is investigated. Both, the imbalance in amplitude and the variation in the phase of the feeding signal are considered.

6.1. Amplitude Imbalance. A variation of the impedances in the differential power divider or resonances in the antenna system could lead to different power levels at both antenna elements. This influence is investigated in this section. It is assumed that the phase difference is exactly $180^{\circ}$. The amplitude balance $\Delta a$ between the feeding signals is defined as

$$
\Delta a=\left.U_{1}\right|_{\mathrm{dB}}-\left.U_{-1}\right|_{\mathrm{dB}} .
$$

To investigate the influence of $\Delta a$ on the array factor, an omnidirectional radiation pattern of the elements is assumed. In this case, (8) can be written as

$$
\begin{aligned}
\underline{\mathbf{A F}}(\theta, \psi)= & {\left[\begin{array}{c}
\mathbf{u}_{\theta} \\
-\mathbf{u}_{\psi}
\end{array}\right] \cdot \Delta a \cdot \frac{1}{\sqrt{2}} e^{j(1 / 2) \beta_{0} d \cos \theta} } \\
& +\left[\begin{array}{c}
\mathbf{u}_{\theta} \\
\mathbf{u}_{\psi}
\end{array}\right] \cdot \frac{1}{\sqrt{2}} e^{-j(1 / 2) \beta_{0} d \cos \theta} .
\end{aligned}
$$

The fixed electrical distance between the elements of $d_{\mathrm{el}}=$ 0.5 is chosen for the investigations (cf. Figure 4(a)). The calculated array factors $\left|\underline{A F}_{\theta, \psi}\right|$ for different $\Delta a$ are shown in Figure 9. The resulting characteristics for varying amplitude balances of $\Delta a=-3 \mathrm{~dB}, \Delta a=-1.93 \mathrm{~dB}$, and $\Delta a=-0.91 \mathrm{~dB}$ are presented in Figures 9(a), 9(b), and 9(c), respectively. The forms of the curves remain essentially the same, however the amplitude of the array factor changes. This is especially visible in the main beam direction, that is, $\theta=90^{\circ}$ for $\left|\mathrm{AF}_{\psi}\right|$. This happens due to nonideal compensation of the crosspolarization radiated from one element by the second one. 


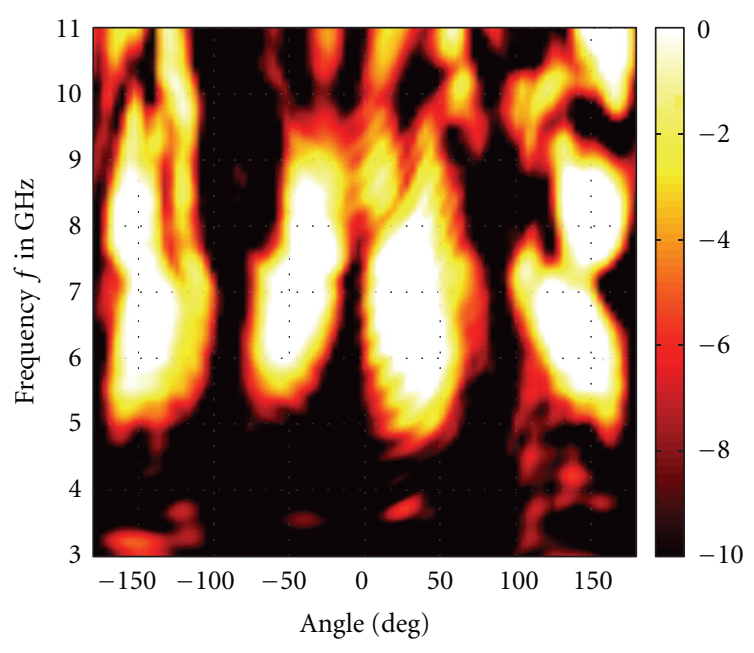

(a) Single antenna, $H$-plane

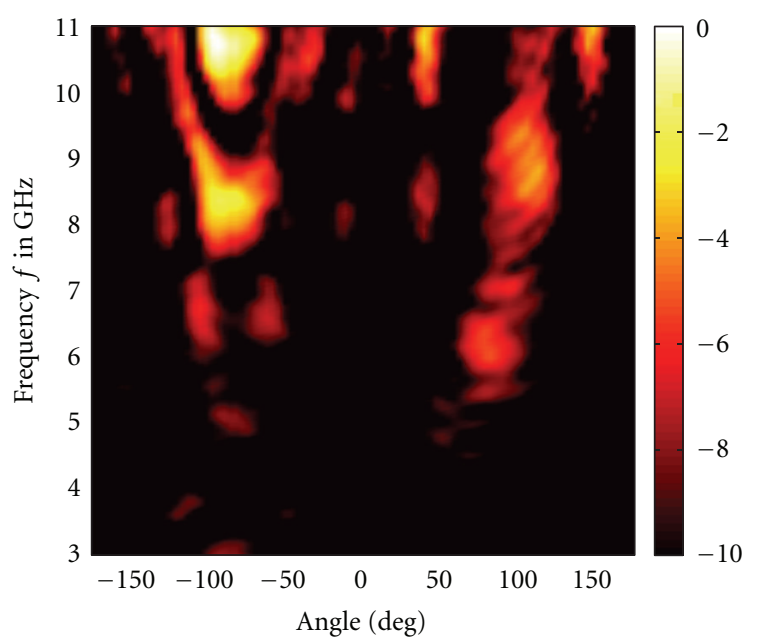

(c) Single antenna, E-plane

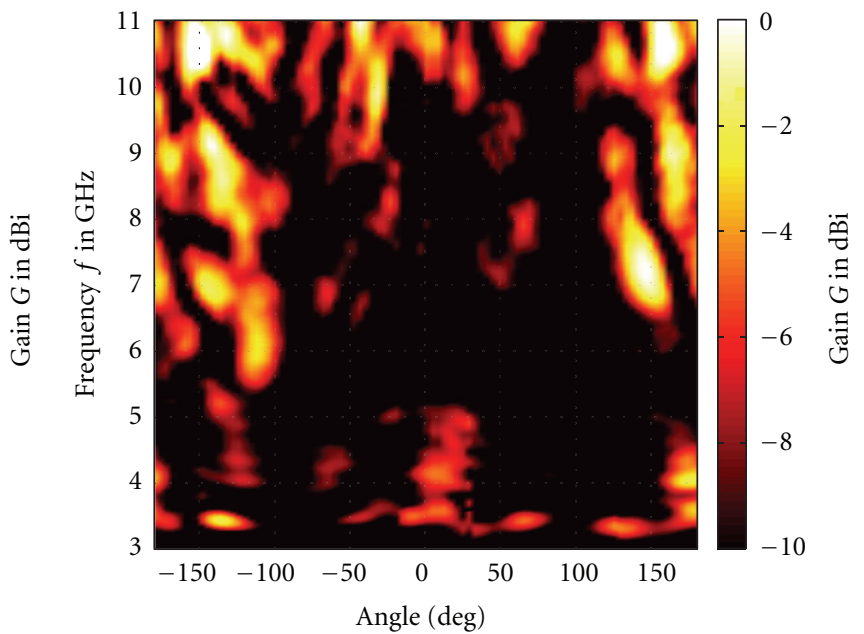

(b) Array, $H$-plane

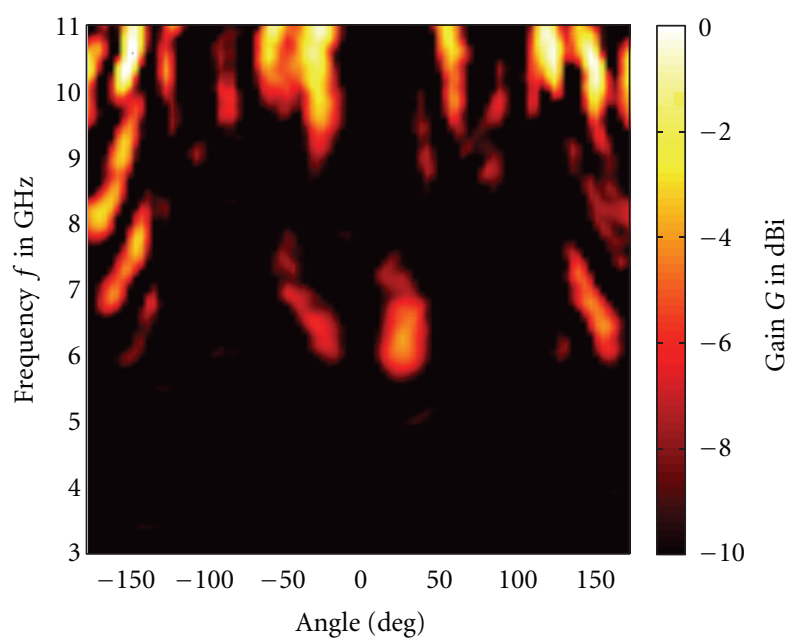

(d) Array, E-plane

FIgURE 8: Measured gain of the single element (Figure 6(b)) and array (Figure 6(d)) in the $X$-polarization, $E$-and $H$-plane over the frequency.

This is a direct influence of the amplitude differences at both of the antennas.

In order to investigate the limits of the principle, the array factor for the cross-polarization $\left|\mathrm{AF}_{\psi}\right|$ at $\theta=90^{\circ}$ is considered. Figure 9(d) shows the dependency of the array factor on the amplitude balance $\Delta a$ at $\theta=90^{\circ}$. If for a proper functionality a value of $-20 \mathrm{~dB}$ is assumed, the maximum amplitude balance has to be below $\Delta a=-1.3 \mathrm{~dB}$, which equals a linear value of 0.86 . In this case, the copolarization is amplified by $2.4 \mathrm{~dB}$ which is $0.6 \mathrm{~dB}$ less compared to the ideal case of identical amplitudes of the feeding voltage.

6.2. Phase Imbalance. The phase of a signal is very sensitive to, for example, coupling effects or resonances and can thus be easily influenced. Furthermore, during the manufacturing process, slight changes in the length of the antenna feeding lines can occur. The influence of these effects is investigated in this section under the assumption that the amplitudes of both signals are identical $(\Delta a=0)$. The phase balance $\Delta \phi$, that is, a deviation from the ideal phase difference of $180^{\circ}$, is defined as

$$
\Delta \phi=\angle\left(\Delta U_{1}\right)-\angle\left(\Delta U_{-1}\right)-\pi
$$

Equation (8) can be written with consideration of $\Delta \phi$ as

$$
\begin{aligned}
\underline{\mathbf{A F}}(\theta, \psi)= & {\left[\begin{array}{c}
\mathbf{u}_{\theta} \\
-\mathbf{u}_{\psi}
\end{array}\right] \cdot \frac{1}{\sqrt{2}} e^{j(1 / 2) \beta_{0} d \cos \theta} \cdot e^{j \Delta \phi} } \\
& +\left[\begin{array}{c}
\mathbf{u}_{\theta} \\
\mathbf{u}_{\psi}
\end{array}\right] \cdot \frac{1}{\sqrt{2}} e^{-j(1 / 2) \beta_{0} d \cos \theta} .
\end{aligned}
$$

Figure 10 shows the array factors $\left|\mathrm{AF}_{\theta, \psi}\right|$ as a function of the phase balance $\Delta \phi$. For a proper comparison, the electrical distance between the elements is again set to $d_{\mathrm{el}}=0.5$. The phase deviation is considered to be $30^{\circ}, 20^{\circ}$, and $10^{\circ}$. The calculated results are shown in Figures 10(a), 10(b), and $10(\mathrm{c})$, respectively. The phase deviation between the feeding signals causes an angular shift of the minima and maxima 


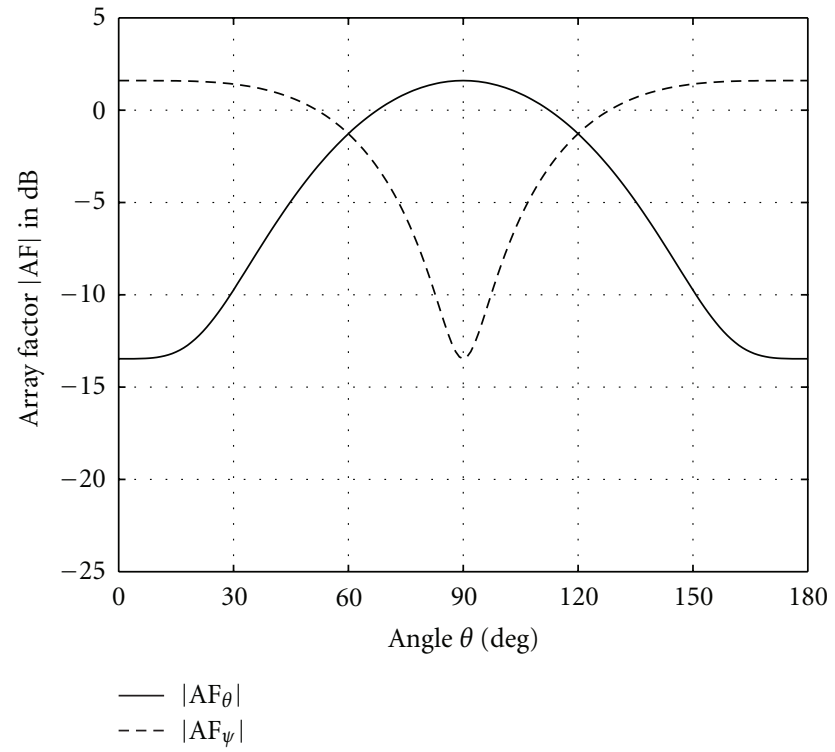

(a) $\Delta a=-3 \mathrm{~dB}$ (linear: 0.7 )

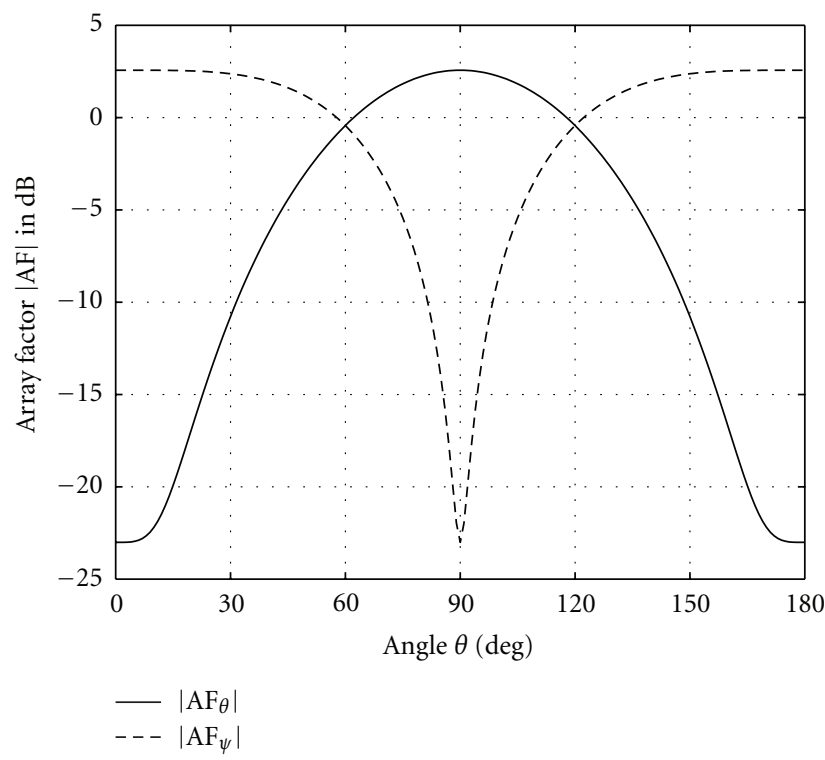

(c) $\Delta a=-0.91 \mathrm{~dB}$ (linear: 0.9 )

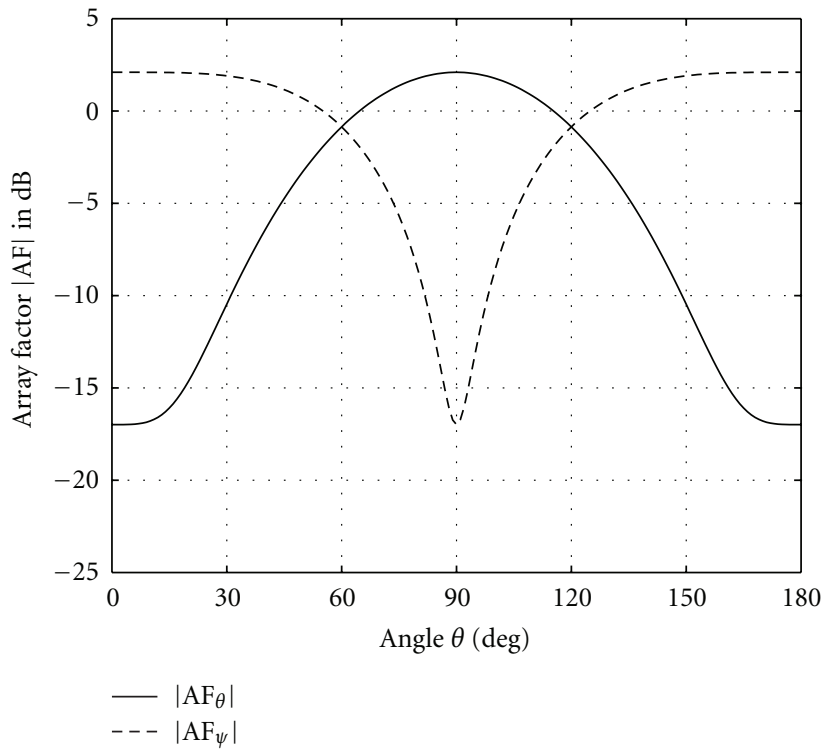

(b) $\Delta a=-1.93 \mathrm{~dB}($ linear: 0.8$)$

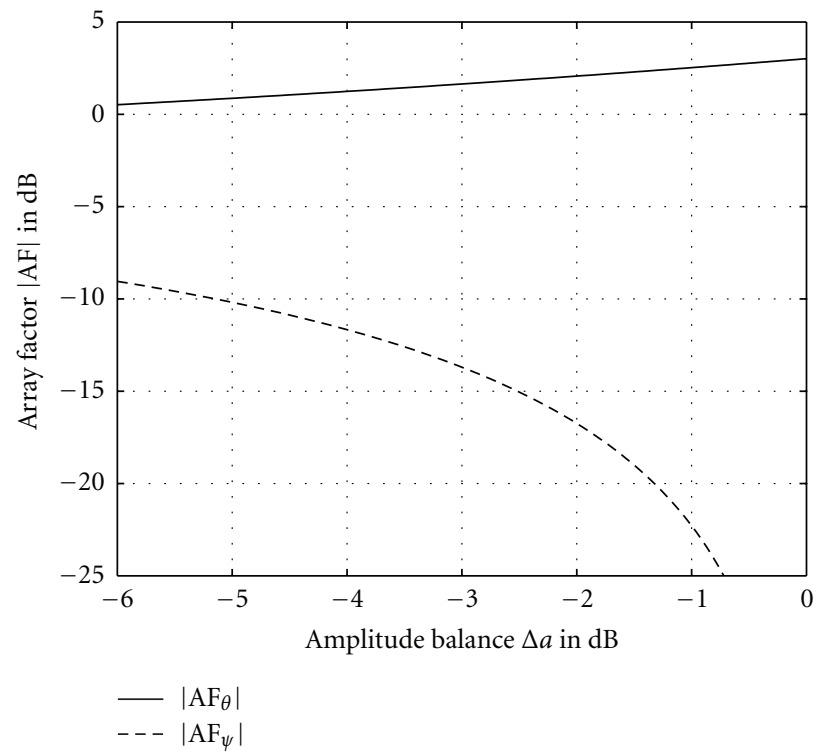

(d) $\left|\mathrm{AF}\left(\theta=90^{\circ}\right)\right|$ in Abhngigkeit von $\Delta a$

Figure 9: Dependency of the array factor on the amplitude balance $\Delta a$ and the angle $\theta$.

of the array factors. The maximal absolute value of the lobes and minimal values of the local minima remain unaffected. At the same time, the patterns are deformed. With increasing phase deviation, grating lobes occur in $\left|\underline{A F}_{\theta}\right|$ in a region around $180^{\circ}$ or $0^{\circ}$, depending on the leading sign of the phase deviation. However, the ability to suppress cross-polarization remains nearly unaffected and acts in the same intensity but at a different angle $\theta$.

If the performance is evaluated in the main beam direction $\left(\theta=90^{\circ}\right)$, a dependency of the array factors $\left|\underline{A F}_{\theta, \psi}\right|$ on the phase balance $\Delta \phi$ can be observed. The dependency is shown in Figure 10(d). If one allows a maximum degradation of suppression of the cross-polarization $\left|\underline{A F}_{\psi}\right|$ to the level of
$-20 \mathrm{~dB}$, the phase imbalance has to be smaller than $8^{\circ}$. For the sake of completeness, it should be noted that a common reason for phase deviations between the feeding, signals is different lengths of the feeding lines. In this case, the phase deviation remains not constant over the frequency but depends linearly on the frequency and the length difference $\Delta l$ of the feeding lines

$$
\Delta \phi(f)=\frac{\Delta l}{\lambda_{r}} 2 \pi=\frac{\Delta l \cdot f}{c_{r}} 2 \pi,
$$

where $\lambda_{r}$ is the wave length in the feeding line and $c_{r}$ the propagation velocity. 


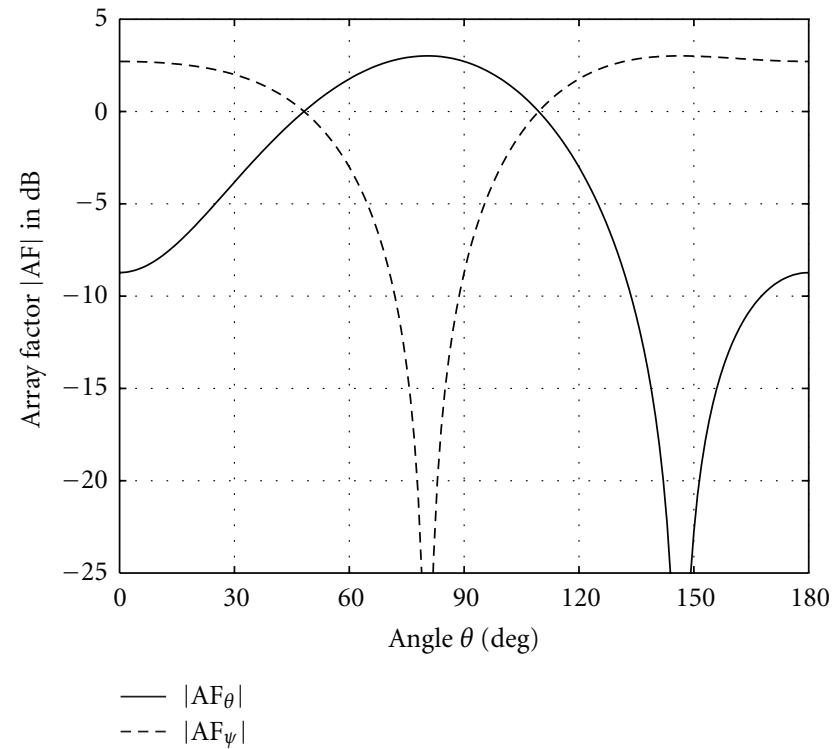

(a) $\Delta \phi=30^{\circ}$

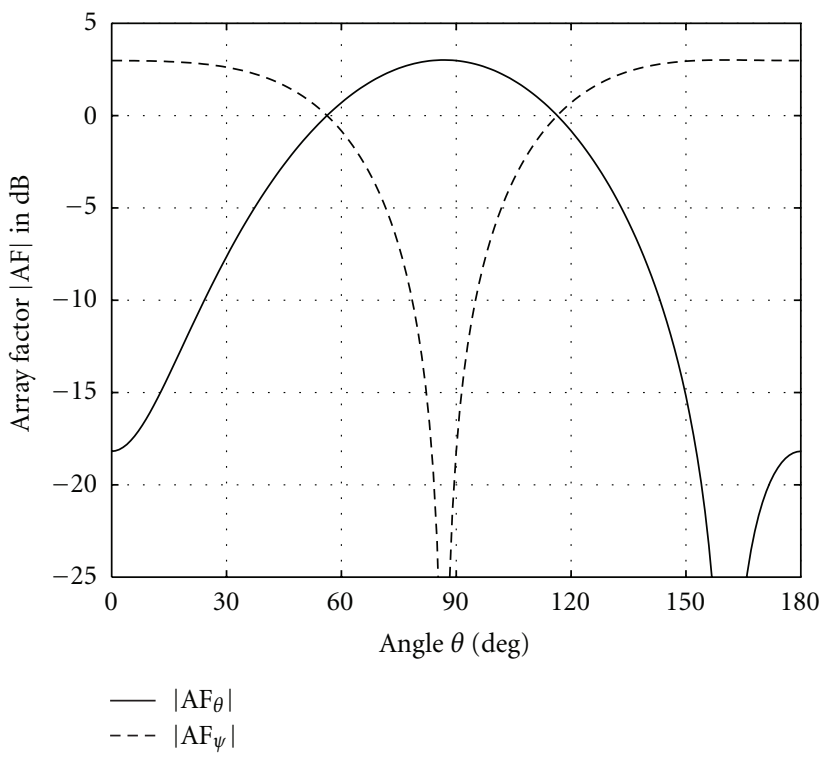

(c) $\Delta \phi=10^{\circ}$

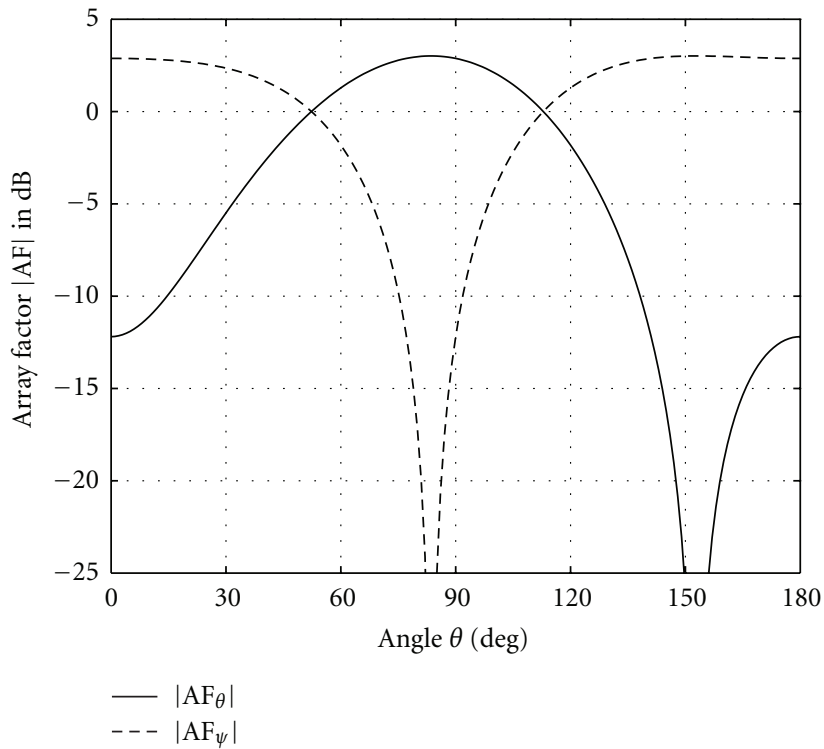

(b) $\Delta \phi=20^{\circ}$

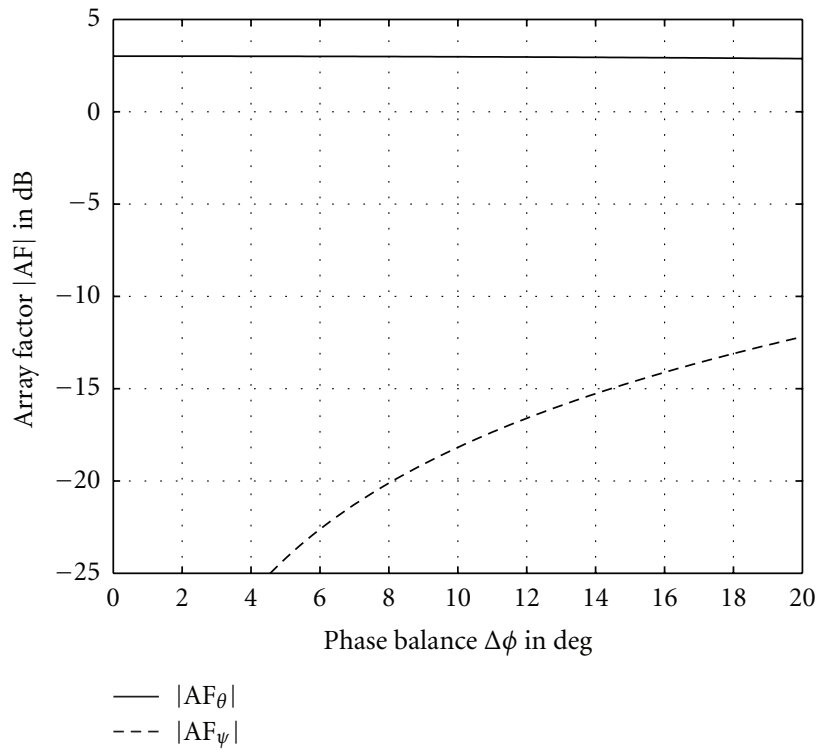

(d) $|\mathrm{AF}|$ depending on $\Delta \phi$

Figure 10: Dependency of the array factor on the phase balance $\Delta \phi$ and the angle $\theta$.

Another issue influencing the performance of the concept is coupling between the array elements. This has been left out of the scope of this paper due to generally low coupling in the presented prototype for verification. The coupling degrades the gain of the whole array but has rather small effect on the polarization suppression, as long as the structure and current distribution in the antenna are kept symmetrical in the desired way. The coupling level, which might be neglected for the proper functionality of the concept, is low enough for most of the planar antennas placed next to each other and possessing the radiation beam perpendicular to the antenna surface. Such antennas are prerequisite for the described applications, nevertheless the designer has to keep the effect of the coupling in mind.

\section{Conclusions}

In this paper, a concept for the realization of fully polarized ultrawideband radiators is introduced. The principle of operation is explained, and a mathematical model for the design of the array is presented. The performance of the configuration regarding the cross-polarization suppression is investigated. The correctness of the considerations has been verified by the measurement of single element and dualpolarized array. The advantage of this configuration is a high polarization purity over a wide angular and frequency range, a very good decoupling between the ports for orthogonal polarizations and a fixed phase center for both polarizations over the entire desired frequency range. The presented 
principle can be applied to all antennas with a main beam direction orthogonal to the substrate surface. The influence of the amplitude and phase balance on the polarization purity is investigated, and the corresponding dependencies are presented.

The solution can be exploited in the design of compact antennas, as presented in $[14,15]$. In these publications an additional advantage is reached, namely, a similarity of the beams in the $E$ - and $H$-plane. The described solution allows for the realization of radiators for high-end radar/imaging systems or high data rate communication devices based on a fusion of MIMO and UWB.

\section{Acknowledgment}

The authors would like to thank the German Research Foundation (DFG) for the financial support.

\section{References}

[1] Federal Communications Commission (FCC), "Revision of part 15 of the commissions rules regarding ultra-wideband transmission systems," First Report and Order, ET Docket 98153, FCC 02-48, 2002.

[2] R. Zetik, J. Sachs, and R. S. Thoma, "UWB short-range radar sensing," IEEE Instrumentation and Measurement Magazine, vol. 10, no. 2, pp. 39-45, 2007.

[3] H. Mott, Polarization in Antennas and Radar, Wiley-Interscience, New York, NY, USA, 1986.

[4] J.-S. Lee and E. Pottier, "Polarimetric radar imaging: from basics to applications," in Optical Science and Engineering, vol. 142, CRC Press, Boca Raton, Fla, USA, 2009.

[5] D. Giuli, "Polarization Diversity in Radars," Proceedings of the IEEE, vol. 74, no. 2, pp. 245-269, 1986.

[6] ETS-Lindgren, 2010, http://www.ets-lindgren.com.

[7] J. Y. Chung and C. C. Chen, "Two-layer dielectric rod antenna," IEEE Transactions on Antennas and Propagation, vol. 56, no. 6, pp. 1541-1547, 2008.

[8] G. Adamiuk, T. Zwick, and W. Wiesbeck, "Dual-orthogonal polarized vivaldi antenna for ultra wideband applications," in Proceedings of the 17th International Conference on Microwaves, Radar and Wireless Communications, vol. 2, pp. 282-285, Wroclaw, Poland, May 2008.

[9] W. Sorgel and W. Wiesbeck, "Influence of the antennas on the ultra-wideband transmission," EURASIP Journal on Applied Signal Processing, vol. 2005, no. 3, pp. 296-305, 2005.

[10] G. Adamiuk, L. Zwirello, L. Reichardt, and T. Zwick, "UWB cross-polarization discrimination with differentially fed, mirrorred antenna elements," in Proceedings of the IEEE International Conference on Ultra-Wideband, (ICUWB '10), Nanjing, China, September 2010.

[11] P. S. Hall, "Dual polarization antenna arrays with sequentially rotated feeding," IEE Proceedings Microwaves, Antennas and Propagation, vol. 139, no. 5, pp. 465-471, 1992.

[12] K. Woelders and J. Granholm, "Cross-polarization and sidelobe suppression in dual linear polarization antenna arrays," IEEE Transactions on Antennas and Propagation, vol. 45, no. 12, pp. 1727-1740, 1997.

[13] J.-Y. Tham, B. L. Ooi, and M. S. Leong, "Diamond-shaped broadband slot antenna," in Proceedings of the IEEE International Workshop on Antenna Technology: Small Antennas and Novel Metamaterials, (IWAT '05), pp. 431-434, March 2005.
[14] G. Adamiuk, W. Wiesbeck, and T. Zwick, "Differential feeding as a concept for the realization of broadband dual-polarized antennas with very high polarization purity," in Proceedings of the IEEE Antennas and Propagation Society International Symposium, (APSURSI '09), pp. 1-4, Charleston, SC, USA, June 2009.

[15] G. Adamiuk, S. Beer, W. Wiesbeck, and T. Zwick, "Dualorthogonal polarized antenna for UWB-IR technology," IEEE Antennas and Wireless Propagation Letters, vol. 8, Article ID 5200399, pp. 981-984, 2009.

[16] G. Adamiuk, L. Zwirello, S. Beer, and T. Zwick, "Omnidirectional, dual-orthogonal, linearly polarized UWB Antenna," in Proceedings of the European Microwave Conference, (EuMC '10), pp. 854-857, Paris, France, September 2010.

[17] G. Adamiuk, J. Timmermann, W. Wiesbeck, and T. Zwick, "A novel concept of a dual-orthogonal polarized ultra wideband antenna for medical applications," in Proceedings of the $3 \mathrm{rd}$ European Conference on Antennas and Propagation, (EuCAP '09), pp. 1860-1863, Berlin, Germany, 2009. 

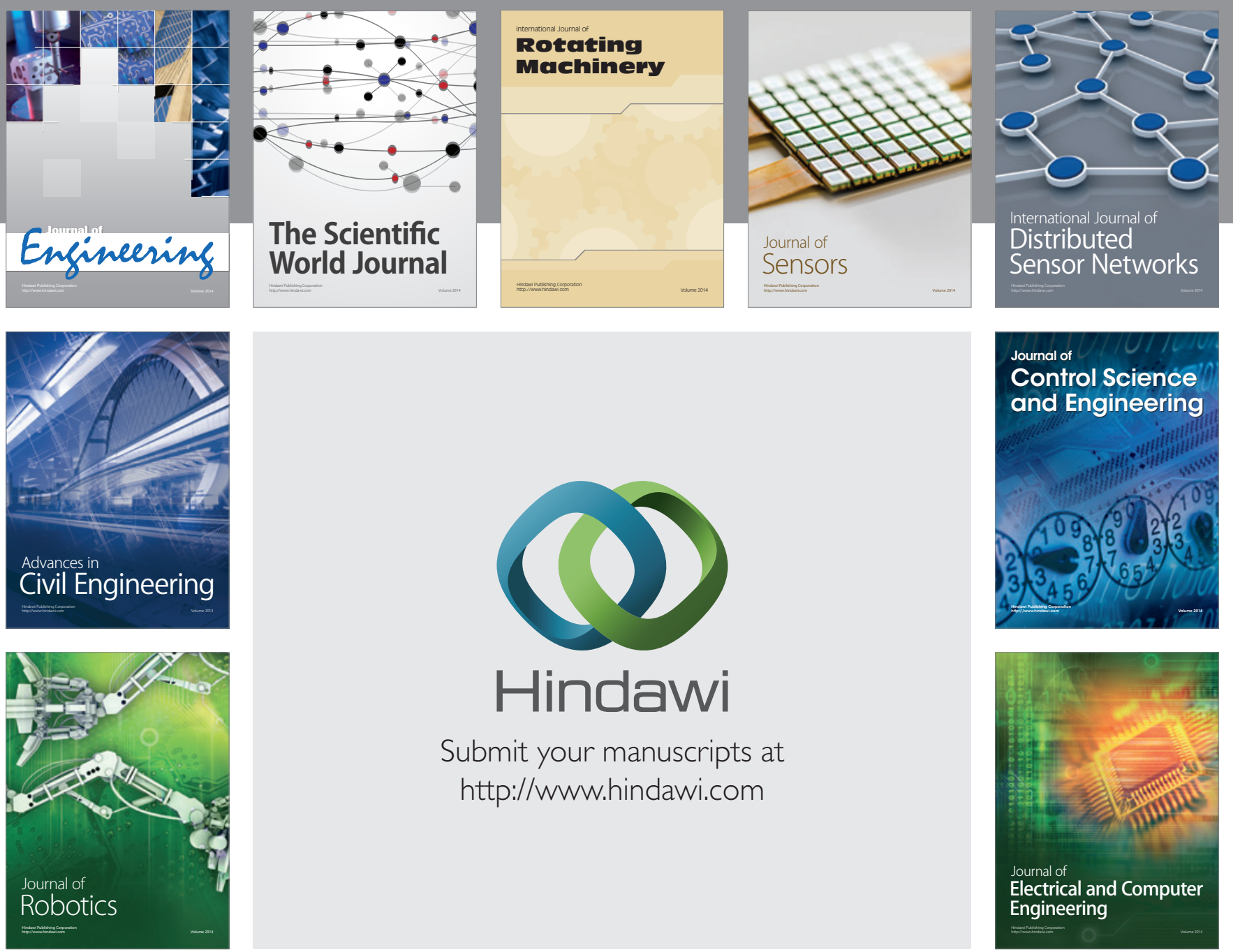

Submit your manuscripts at

http://www.hindawi.com
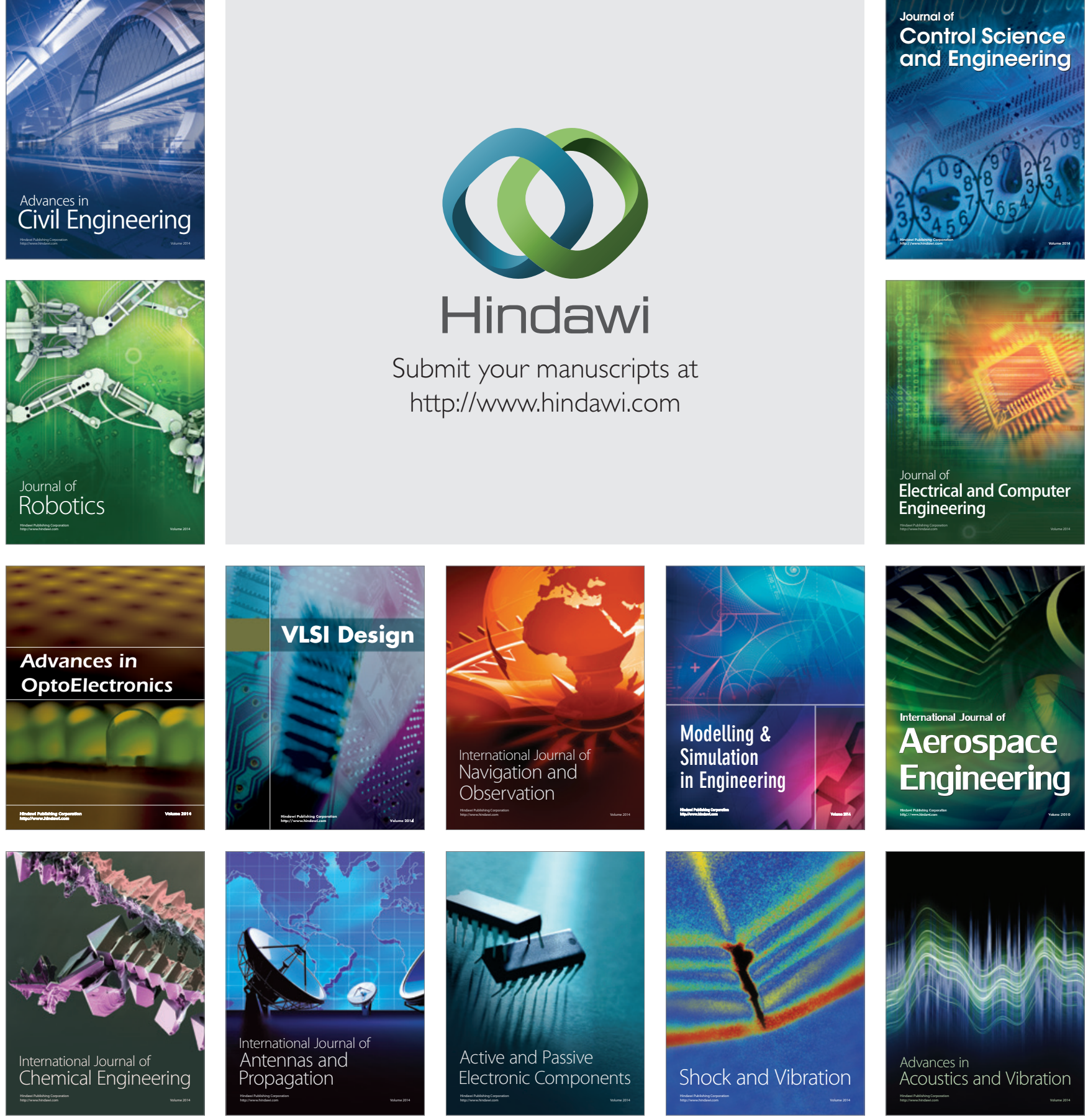